\title{
Breve cartografía de la educación popular: enfoques entre Brasil-Colombia
}

\author{
VÉLEZ, Carlos Guillermo Mojica ${ }^{1}$ \\ CASTRO, Amanda Motta
}

\section{RESUMEN}

Este es un trabajo teórico realizado durante el programa de Maestría en Educación de la Universidad Federal de Río Grande / FURG. El objetivo fue analizar teoricamente lo que se ha producido sobre los preuniversitarios populares (PP) en Brasil y en Colombia, especialmente en la ciudad de Medellín, durante los últimos 5 años. Históricamente, Brasil ha hecho una contribución significativa a América Latina y el Caribe al pensar en una educación gratuita, abierta, democrática y de puertas abiertas para todos y todas los que quieran estudiar. Esta es una lucha que aún continúa y para lograrla se han realizado esfuerzos teóricos y militantes en varios países, entre ellos Colombia con los preuniversitarios populares. La cartografía presentada aquí demuestra cuánto la investigación militante puede generar voces de esperanza para la trasnformación de las universidades y las desigualdes sociales en dos países diferentes de América Latina.

\section{Educacion Popular. Preuniversitarios Populares.}

\section{Brief cartography of popular education: approaches between Brazil- Colombia}

\section{ABSTRACT}

This is a theoretical work carried out during the Master's in Education program of the Federal University of Rio Grande / FURG. The objective was to analyze what has been produced about popular pre-university course (PP) in Brazil and Colombia, especially in the city of Medellín, during the last 5 years. Historically, Brazil has made a significant contribution to Latin America and the Caribbean by thinking about a free, open, democratic and open-door education for everyone who wants to study. This is a struggle that still continues and to achieve it, theoretical and militant efforts have been made in several countries, including Colombia with the popular pre-university course. The cartography presented here

\footnotetext{
${ }_{1}^{1}$ Mestrando em Educação pela Universidade Federal do Rio Grande. Colômbia. E-mail: carlosmojv@gmail.com. Lattes: http://lattes.cnpq.br/4416694608599759. ORCID: https://orcid.org/0000-0003-4015-1607.

2 Doutora pelo programa de Pós-Graduação em Educação da Universidade do Vale do Rio dos Sinos/UNISINOS. Professora do Programa de Pós-Graduação em Educação da Universidade Federal do Rio Grande do Sul/FURG e docente do Departamento de Educação da mesma instituição. Brasil. E-mail: motta.amanda@gmail.com. Lattes: http://lattes.cnpq.br/3449473426395924. ORCID: https://orcid.org/0000-0003-04711240.
} 
demonstrates how much militant research can generate voices of hope for the transformation of universities and social inequalities in two different countries in Latin America.

Popular Education. Popular Pre-university Course.

\section{Breve Cartografía de la Educación Popular: enfoques entre Brasil- Colombia}

\section{RESUMO}

Este é um trabalho teórico realizado durante o programa de Mestrado em Educação da Universidade Federal de Río Grande / FURG. O objetivo foi analisar teoricamente o que ocorreu sobre a pré-universidade popular ( $P P$ ) no Brasil e na Colômbia, especialmente na cidade de Medellín, nos últimos 5 anos. Historicamente, o Brasil fez uma contribuição significativa para a América Latina e o Caribe ao pensar em uma educação gratuita, aberta, democrática e de portas abertas para todos que desejam estudar. Esta é uma luta que ainda continua e para alcançá-la, esforços teóricos e militantes foram feitos em vários países, incluindo a Colômbia, com as pré-universidades populares. A cartografia apresentada aqui mostra quanta pesquisa militante pode gerar vozes de esperança para a transformação de universidades e desigualdades sociais em dois países diferentes da América Latina.

Educacion Popular. Preuniversitarios populares.

\section{Breve cartografia dell'educazione popolare: approcci tra Brasile-Colombia}

\section{SINTESE}

Questo è un lavoro teorico svolto durante il programma di Master of Education dell'Università Federale del Rio Grande / FURG. L'obiettivo era di analizzare teoricamente ciò che è stato prodotto sui popolari studenti pre-universitari (PP) in Brasile e Colombia, in particolare nella città di Medellín, negli ultimi 5 anni. Storicamente, il Brasile ha dato un contributo significativo all'America Latina e ai Caraibi pensando a un'istruzione libera, aperta, democratica e aperta a tutti coloro che vogliono studiare. Questa è una lotta che continua ancora e per raggiungerla, sono stati fatti sforzi teorici e militanti in diversi paesi, tra cui la Colombia con i popolari studenti pre-universitari. La cartografia presentata qui dimostra quanto la ricerca militante possa generare voci di speranza per la trasformazione delle università e le disuguaglianze sociali in due diversi paesi dell'America Latina.

Educazione popolare. Pre-università popolare. 


\section{Primeras palabras...}

Tanto mais democrática uma universidade quanto mais tolerante, quanto mais se abre a compreensão dos diferentes, quanto mais se pode tornar objeto da compressão dos demais.

Paulo Freire

Las investigaciones en torno a las relaciones entre educación popular (EP) y educación superior, y específicamente sobre los preuniversitarios populares (PP), no es un tema nuevo de conocimiento lo que se muestra en este trabajo. Aunque las producciones académicas se concentran en países como Brasil, Argentina, México y Cuba, numerosas experiencias se reportan en Ecuador, Perú, Venezuela, Chile, Colombia, entre otros.

Por lo anterior, en este apartado se expondrá cuáles son los principales puntos de análisis, metodologías de estudio y conclusiones que las investigaciones recientes en el tema reportan durante los últimos 5 años. Para ello, en primer lugar, se explicará cómo se desarrolló la búsqueda de información bibliográfica y se realizará una mirada panorámica sobre sus estudios.

Posteriormente, se abordará los logros, desafíos y características de estos espacios de formación, después se explicará los conceptos y prácticas en torno a las universidades populares para finalmente mostrar los resultados de investigaciones concretamente en Colombia y la ciudad de Medellín.

\section{Educación popular y educación superior: los preuniversitarios populares como objetos de investigación}

Para la búsqueda de información se definieron las siguientes palabras claves: educación popular, educación superior, acceso y práctica. Despues, a medida que se encontraron documentos que no respondieron a los intereses de investigación, dichas palabras claves fueron cambiadas por dos centrales, preuniversitarios populares y prácticas. Ahora bien, con esas palabras se realizaron ecuaciones de búsqueda. En la siguiente tabla se describe las operaciones realizadas:

Tabla 1. Metodologia búsqueda de información

Ecuación de búsqueda
1. (educación popular) AND (educación superior OR universidad) and (acceso) and (prácticas)

2. (Preuniversitario popular) AND (prácticas) NOT (educación de adultos). 


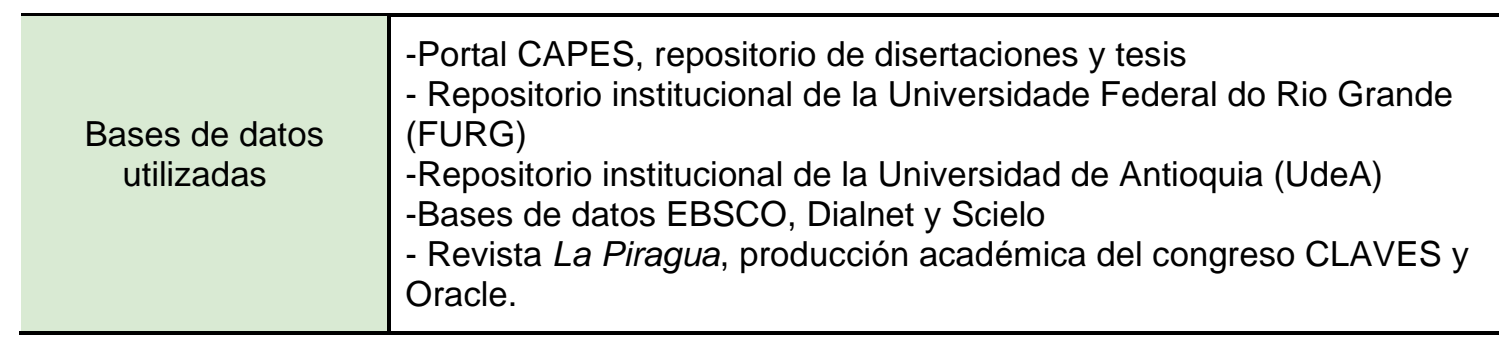

Fuente: construcción propia.

En este proceso de búsqueda se usaron únicamente resultados de Colombia y Brasil. En Argentina, Chile, México y otros países de la región existen otro conjunto amplio de investigaciones pero que sobrepasan los límites de este trabajo. Sin embargo, esto muestra que los PP no son un proceso educativo reducido a un único país, lo que podría ser interesante para futuros estudios en un nivel macro latinoamericano. Se ha privilegiado Colombia ya que es el contexto de investigación, y Brasil por ser el país con mayor trayectoria de estas experiencias en América Latina (en el portal de la CAPES aparecen más de 80.000 investigaciones en relación a los PP), y además es la cuna de la educación popular freiriana ${ }^{3}$.

En las investigaciones se evidencia multiples interpretaciones de lo que es la EP como: (I) una metodología y didáctica de la educación que articula la enseñanza de contenidos disciplinares con la formación política y ciudadana; (II) proyectos comunitarios para la formación de los movimientos sociales; (III) conjunto de prácticas más o menos estables y articuladas de acuerdo a principios políticos y educativos; (IV) y en otros trabajos como modelo pedagógico de movimientos sociales latinoamericanos. En esta amplitud, la mayoría de las investigaciones son estudios de caso y algunas veces comparados, puesto que su lugar de actuación y los sujetos que la realizan cambia de uno a otro.

De acuerdo con lo anterior, ¿qué son los preuniversitarios populares? y ¿se podrían definir como movimientos sociales, organizaciones estudiantiles 0 cursos preparatorios? a lo largo de los documentos se identifican varias líneas y sentidos de tensión, algunas veces convergentes para responder a la pregunta por el qué son los PP.

Para comenzar, Magalhaes (2018) analiza desde la perspectiva de la sociología de la educación las diferencias entre los preuniversitarios populares a los convencionales. En general, la autora señala que los cursos preparatorios para la universidad existen desde la creación misma de estas instituciones en América Latina, especialmente cuando se desarrollan pruebas selectivas de acceso, y se crea la necesidad de fortalecer la formación de los estudiantes que ingresan a la educación superior. No obstante, a finales del siglo XX se comienza

\footnotetext{
${ }^{3}$ En Brasil, los preuniversitarios populares tienen diferentes nominaciones como: pre-
} vestibulares populares, cursinho popular, curso pre-universitario popular, entre otros. 
a ser más fuerte y visible en Brasil, y posteriormente en otros países de la región, cursos con una metodología y principios diferentes a los tradicionales, los cuales se conocen como los preuniversitarios populares (PP).

Estos PP se diferencian de los convencionales en la medida que denuncian las inequidades educativas y sociales del sistema, el cual está construido desde y para la exclusión de una gran parte de la población, con foco hacia los colectivos vulnerables, grupos históricamente excluidos y las clases populares y trabajadoras. Esto quiere decir que, la prueba del examen de admisión a la Universidad no es simplemente una prueba, sino un mecanismo más de exclusión del derecho humano a la educación. Por esto, "não é possível considerar a aprovação apenas como uma questão de mérito, sem levar em conta a formação/origem dos sujeitos que disputam os exames de seleção" (MAGALHAES, 2018, p. 24). En los PP se hace una crítica radical a la meritocracia como una ideología estructural de la sociedad.

Al coincidir con Bourdeau, Magalhaes muestra cómo las diferencias entre la educación pública con la privada, la escuela rural a la urbana y las distintas herencias culturales de las familias, sumada a la condición económica hace que los estudiantes compitan en condiciones injustas, ya que desde muy temprano los jóvenes están limitados por las desigualdades sociales que los condenan a ser rechazados por las universidades. En pocas palabras, no se puede entender el acceso a la universidad como una prueba de contenidos, sino como una herramienta de poder en la cual se ponen en juego los capitales sociales de un individuo basados en la lógica de la meritocracia. De este modo, perder una prueba de admisión no es el resultado de la inteligencia o la capacidad, sino un fracaso estructural que evidencia situaciones de desigualdad cognitiva, en la medida que la meritocracia no es una ética de lo público, sino un resultado del individualismo moderno y capitalista, como afirma Magalhaes.

En consecuencia, los PP surgen como resistencia a un modelo de universidad elitista y meritocrática. Estos espacios son diferentes en conmparación con los tradicionales dado que no suelen cobrar inscripción, sus profesores y coordinadores son voluntarios y/o militantes de los proyectos, y los PP son apoyados por distintos sindicatos de estudiantes y maestros o por movimientos sociales y políticos. Las personas que por lo general acceden a estos preparatorios son estudiantes trabajadores y de las periferias de las grandes ciudades, además que las clases se desarrollan en espacios cedidos por instituciones públicas o privadas sin tener una sede propia generalmente.

Adicionalmente, se trasciende la enseñanza de contenidos para abordar distintos temas éticos y políticos con el fin de comprender críticamente cómo funciona el sistema que los hace fracasar, y los distintos modos de jugar y resistir a él. Por lo tanto, se busca ampliar los capitales de los estudiantes y que ellos tengan más confianza y autoestima para acceder y permanecer en las universidades, basados en los principios de la educación popular. 
Cabe destacar que, la educación popular (EP) no puede ser entendida como un concepto, metodología o movimiento pedagógico cerrado, sino que es flexible y dinámico. Esto significa que existen diversas comprensiones sobre lo que es la educación popular, y por eso mismo los PP son diversos. En los 39 documentos que constituyen el trabajo es posible advertir ello: cada PP es un modo de hacer y pensar la educación popular diferente por sus contextos particulares de acción. No obstante, todos ellos comparten búsquedas similares, algunos más cercanos a Freire y otros solo como una referencia o inspiración, pero que en todos los casos es la columna conceptual de los preuniversitarios.

En este sentido, una posible definición que abarque y caracterice a a los PP es que ellos son

Ações de democratização do acesso ao ensino e de construção do saber com as classes populares. Além disso, tais cursos podem ser interpretados como redes de indignação e esperança, em função de seu caráter social de subversão e luta contra o sistema educacional [...] com conexões em rede estabelecidas para articular mentes, criar significado e contestar o poder. (COSTA YGÓMEZ, 2017, p. 137).

La indignación se refiere precisamente al carácter de denuncia explicado anteriormente, es decir, a la situación de injusticia social en los exámenes de acceso a la universidad; y la esperanza es leída en el sentido freiriano. Por ello, la esperanza no se entiende en este contexto como el acto de esperar o una virtud religiosa, y tampoco un valor positivo de las personas, sino como el deseo de transformar el mundo, deseo que se vuelve resistencia y que "assim esperança como uma reivindicação à transformação da realidade" (p. 141). Por último, es importante subrayar la palabra redes. Los PP por su cercanía a movimientos sociales y su carácter de educación popular basados en principios éticos y políticos se caracteriza por realizarse siempre en colectivo hacia otros colectivos (MAGALHAES, 2018).

Por su parte, Almeida (2016) señala que los PP son respuestas sociales de los jóvenes frente a la ausencia del Estado. En otras palabras, como el Estado no es capaz de garantir el derecho a la educación superior para las clases populares los PP responden a ese derecho parcialmente, trabajando para alcanzar el acceso. De igual manera, estos preuniversitarios están donde precisamente la universidad no está: sus voluntarios y militantes van a las periferias de las ciudades para encontrar allí una gran cantidad de jóvenes y adultos que se sienten lejanos a la universidad, "Deste modo, os PréVestibulares Populares precisam ser compreendidos na tensão inclusãoexclusão dos excedentes das seleções de ingresso nas universidades e dentro das contradições das políticas de acesso à educação superior" (p. 111). 
Almeida, muestra que los PyP surgen en Brasil especialmente con los movimientos negros, y que ellos se vuelven actores claves para discutir las políticas afirmativas y de Estado en educación superior, por ejemplo, sobre las cuotas para indígenas y quilombos en las universidades. Los PP son realizados por múltiples agentes con perspectivas políticas que no siempre coinciden, algunos incluso son acciones institucionalizadas de las universidades 0 proyectos de extensión. En este contexto, Almeida realiza un panorama sobre las temáticas y metodologías que se investigan sobre los PP en Brasil. A continuación, se resumen sus hallazgos con palabras claves:

Tabla 1: temas de investigación sobre los PP en Brasil

\begin{tabular}{l|l}
\hline Tema & Descripción \\
\hline $\begin{array}{l}\text { Emergencia y construcción de } \\
\text { los PP. }\end{array}$ & $\begin{array}{l}\text { Se describe y analiza la historia y los procesos por los } \\
\text { que nacen diferentes PP y sus contextos educativos. }\end{array}$ \\
\hline Formas organizativas de los PP. & $\begin{array}{l}\text { Se estudian las formas de autogestión y elaboración de } \\
\text { mapas sociales de los PP. Además, como ellos } \\
\text { conversan con las políticas educativas. }\end{array}$ \\
\hline $\begin{array}{l}\text { Relaciones de enseñanza y } \\
\text { pedagógicos de los PP. }\end{array}$ & $\begin{array}{l}\text { Se estudian las prácticas de enseñanza y los } \\
\text { aprendizajes, desde una mirada didáctica y curricular. }\end{array}$ \\
\hline $\begin{array}{l}\text { Políticas públicas de acceso a la } \\
\text { educación superior. }\end{array}$ & $\begin{array}{l}\text { Se investiga conceptos como equidad y acción afirmativa. } \\
\text { Los PP como actores políticos, se preguntan por los } \\
\text { impactos de las políticas. }\end{array}$ \\
\hline $\begin{array}{l}\text { El carácter educativo-político de } \\
\text { los cursos. }\end{array}$ & $\begin{array}{l}\text { Indagan por la formación política de los PP y los distintos } \\
\text { sentidos de la educación popular a través de las } \\
\text { trayectorias militantes de sus miembros. }\end{array}$ \\
\hline $\begin{array}{l}\text { Teorías de acción colectiva de } \\
\text { los movimientos sociales. }\end{array}$ & $\begin{array}{l}\text { Investiga los PP como movimientos sociales urbanos. } \\
\text { Problematiza los conceptos de democracia y ciudadanía. }\end{array}$ \\
\hline & $\begin{array}{l}\text { Se analizan las aspiraciones, formación y clasificación de } \\
\text { los estudiantes. }\end{array}$ \\
\hline
\end{tabular}

Fuente: construcción propia, a partir de Almeida (2016).

Teniendo en cuenta esta tabla que muestra en líneas generales las tendencias de investigación, a continuación, se detallan los hallazgos más importantes.

\section{Los PP: sus logros, desafíos y características}

¿Cómo una propuesta de educación popular se materializa en las prácticas pedagógicas de un preuniversitario hecho por jóvenes y para jóvenes?, 
¿de qué modo se puede articular la formación ética y política -que habla Freirecon la preparación a un examen de contenidos académicos, en un contexto profundo de desigualdad social y donde predomina meritocracia?

En las investigaciones hay tres caminos metodológicos para hacerlo: la primera, -la más común- es con la creación de asignaturas alternativas a las tradicionales, agregando así contenidos de carácter político y sociocultural al currículum, por ejemplo, se crea la disciplina de racismo y procesos de exclusión, formación ciudadana, lecturas críticas de contexto, además de las disciplinas tradicionales como matemáticas, física, biología, etc; en otros casos se hacen proyectos que transversalizan a todas las áreas, donde se articulan profesores para un abordaje más crítico y social de los contenidos, seleccionando temas centrales para todos y todas; y por último, hay experiencias donde se relacionan la formación académica y política a través de las prácticas cotidianas de sus maestros en la enseñanza de los contenidos mismos de las pruebas. Es decir, no se crean nuevas materias, sino que cada disciplina se desarrolla bajo una metodología que posibilita la formación ética y política, y que puede - o no- tener proyectos transversales. Las investigaciones más recientes se inclinan a la tercera opción metodológica, ya que se muestran propuestas donde lo político está en la enseñanza misma de los contenidos.

Al respecto, Sousa (2015) problematiza la enseñanza de la historia desde la EP en los PP y la educación media, a través de un estudio de caso en Paraíba. Él muestra caminos posibles para enseñar historia universal, historia de Brasil e historial local articulado a la historia personal de los estudiantes. No se trata de enseñar niveles distintos de la historia o historias diferentes, sino historias relacionadas, en donde una contiene a las otras con múltiples puntos de conexión.

En este sentido, la EP como modelo pedagógico y marco metodológico permite la articulación de contenidos con la realidad cercana de los estudiantes, especialmente a través del carácter dialógico de la EP. Así pues, la historia no es concebida como una línea recta de acontecimientos que necesitan ser memorizados, sino como puntos de conexión del pasado -incluso antiguo- con la realidad presente de cada estudiante, y que trae consigo una reflexión política y ética del mundo. No obstante, Sousa reconoce las limitaciones que existen para lograr este propósito, ya que el tiempo es reducido, los grupos de estudiantes son muy heterogéneos en edades, intereses y aprendizajes y la formación de los profesores necesita fortalecerse aún más.

Desde esta perspectiva, Vasconcelos (2015), explora a través de la enseñanza de la geografía en los PP los dilemas pedagógicos más comunes a los que se enfrentan los profesores, especialmente sobre cómo relacionar la formación política con la académica, con miras al examen de admisión a la universidad. El autor, el cual hace un estudio de caso múltiple en Niteroi, señala que los conceptos freirianos de educación bancaria y emancipatoria son 
herramientas para transformar la práctica pedagógica, logrando superar el simple contenido de la prueba de un modo bancario por otro que sea emancipador.

Tanto Sousa como Vasconcelos resaltan la importancia del carácter dialógico de la educación popular, en la medida que es a través del diálogo que se le da un sentido político a lo educativo, y que ese mismo sentido es emancipador. Cabe resaltar que, la dialogicidad no se trata únicamente de hacer las clases más participativas o con métodos como juegos interactivos, sino de poner a conversar a los contenidos con los estudiantes y los profesores desde una perspectiva problematizadora. En consecuencia, los estudiantes al conversar con otros estudiantes, con distintos profesores y con múltiples maneras de ver los contenidos se ven llamados a cambiar su mirada, para que ella se amplíe y politice

Ahora bien, al igual que Sousa, Vasconcelos se preocupa por la formación de los profesores de los PyP para desarrollar la dialogicidad como metodología en educación. El investigador explica que la dialogicidad en cada PyP es diferente, de acuerdo con los principios e identidades que cada grupo de jóvenes construye

Os PVPs constituem um movimento em que a reposição de identidades é constante, as identidades são fluidas assim como as próprias agendas dos movimentos, que como vimos no item anterior se alteram de acordo com as dinâmicas interna e externa do movimento, o indivíduo constrói sua identidade junto com o movimento. (SOUSA, p. 44).

El investigador analiza las prácticas educativas de los PyP considerando a estos grupos como movimientos sociales juveniles. Para él, cada movimiento tiene prácticas pedagógicas distintas, un movimiento social es también un movimiento educativo de acuerdo a la militancia e ideología de sus miembros, por eso dichas prácticas corresponden a las identidades políticas de sus miembros. Sin embargo, los PyP están conformados por diversas identidades de corrientes políticas diferentes, algunos vinculados a movimientos negros, indígenas, feministas, de trabajadores, incluso católicos y de pastorales sociales, entre otros; por lo tanto, las prácticas como las identidades son fluidas y no son estáticas. Es decir, cuando se estudia los procesos de enseñanza y aprendizaje de los PyP no se puede hacerlo como una metodología estática, sino que, al contrario, es múltiple y diversa, y por lo tanto creativa en muchas de sus acciones, debido al carácter cambiante del movimiento y las identidades de los jóvenes.

La educación popular en este caso no es para los PP un paso-paso que guía metodológicamente la enseñanza de algún objeto de conocimiento, sino más bien un camino para problematizar y orientar el abordaje de contenidos de 
una manera crítica y dialógica. Un ejemplo de ello lo presenta Dos Santos (2017) cuando investiga la enseña de la química en los PP de la ciudad de Pelotas (RS)

\begin{abstract}
Alguns assuntos tratados nas Situações de Estudo, como os efeitos da poluição atmosférica para o ambiente e a saúde, ou a leitura de bulas de medicamentos no estudo de soluções, considerando a abordagem fenomenológica, representacional e teórica dos conhecimentos químicos, foram importantes para que os alunos refletissem sobre o papel do conhecimento científico nas suas vidas. (p. 144).
\end{abstract}

O Ramos (2018), respecto a la alfabetización en inglés en un PP ubicado en el Estado do Rio de Janeiro, en la ciudad de São Gonçalo

Aqui temos, então, o ensino de uma língua estrangeira como espaço de fronteira: como instrumento que pode ser usado não só em prol de práticas coloniais, mas também como possibilidade para respostas decoloniais, para outra globalização. (p. 41).

Tanto en el caso de la química como en el del inglés se evidencia varios elementos característicos en común. En primer lugar, los esfuerzos por poner en diálogo los contenidos de las pruebas con conocimientos para la formación ciudadana y política, alrededor del mundo cotidiano del estudiante. En segundo lugar, este esfuerzo surge por la militancia de sus jóvenes profesores como miembros activos de movimientos sociales.

El inglés como la química, historia y geografía son vistos como dispositivos de poder, dispositivos en cuanto a que, estos conocimientos si bien pueden ser barreras de las desigualdades cognitivas de los estudiantes para acceder a la educación superior, también pueden ser herramientas para situarse y comprender mejor el mundo, y para, en definitiva, resistir y responder a las injusticias sociales. En el caso de la química se genera una mirada crítica frente a la alimentación y el aire para criticar la lógica de la producción capitalista, y en el inglés una comprensión más amplia de los significados sociales y culturales de los idiomas. De este modo, no se aprende el inglés por ser la lengua del desarrollo del primer mundo, sino como una necesidad para comprender la cultura del otro y ponerla en diálogo con la propia. Por otro lado, la química no se enseña como un conjunto de fórmulas o teorías, sino que se articula a problemas socioambientales que se unen a la agenda de movimientos jóvenes ambientalistas, veganos y de protección animal.

Ahora bien, Ramos trae aportes significativos al relacionar la multiculturalidad e interculturalidad a la EP, él explica que la educación popular es una educación que reconoce las diversidades de los estudiantes, y hace de esa diversidad un tema constante de investigación y articulación. Por otra parte, Dos Santos hace énfasis en la formación de los profesores para relacionar la 
química de los exámenes con la química de lo cotidiano y dice que los principios de la democracia y transformación social que orienta la EP es el marco fundamental de trabajo de todas las propuestas.

Estas propuestas didáctico-pedagógicas enfrentan diversos desafíos como señala Pereira y Gonçalves (2012), en cuanto a que lograr una formación crítica y emancipatoria con una visión solidaria y colectiva que se propone desde la EP no es una tarea fácil en los PP.

Estos espacios se enfrentan a desafíos tanto estructurales como otros propios de cada contexto. Algunos desafíos estructurales son la meritocracia, la gestión de una universidad elitista y la burocracia del sistema, y otros propios del contexto y que ocurren frecuentemente son:

- Las distancias físicas que los estudiantes tienen para asistir a las clases, los estudiantes viven muy lejos y no cuentan con los recursos para llegar a los encuentros.

- La realización y adquisición de material pedagógico, considerando la condición de pobreza y exclusión social de la mayoría de sus miembros.

- El poco tiempo para el estudio bajo la presión social y personal de aprobar un examen de admisión.

- La falta de formación pedagógica de los profesores de los PP, en la medida que la mayoría de ellos son jóvenes en formación aún no profesionales.

A lo anterior, se le puede sumar: la falta de sostenibilidad para los profesores y estudiantes que asisten, en la medida que sus maestros suelen hacer ejercicio voluntario y los estudiantes no tienen apoyo económico para asistir; la rotatividad de los maestros, ya que muchos entran y salen de los PP dejando a medias procesos educativos; la heterogeneidad de los estudiantes y la falta de condiciones básicas relativas a la infraestructura y materiales.

Sin embargo, Pereira y Gonçalves menciona que, si bien la educación popular y los PP no están exentos de incoherencias y equivocaciones, sus prácticas pueden ser pensadas como laboratorios de experiencias pedagógicas para la formación de maestros iniciantes. En otras palabras, laboratorios pedagógicos de jóvenes en formación que muestran otra forma de hacer educación posible basada en principios de la solidaridad y el pensamiento crítico, y que devela las esperanzas y utopías del acceso de las clases populares y las contradicciones de las políticas de acceso a la universidad.

Así como la dialogicidad es un concepto fundamental, también lo es el de la solidaridad. En todos los 39 documentos que hacen parte de este estado de arte se resalta que una característica de éxito de estas experiencias es el enlace comunitario y las relaciones de amistad que se generan entre sus miembros. Esta solidaridad hace que los lazos entre profesores y estudiantes sean más horizontales que en la educación tradicional, e incluso es común que los profesores sean amigos entre ellos, y que los estudiantes de los PP se vuelvan 
profesores en el futuro de los mismos PP en los que estudiaron. Los jóvenes, tanto maestros como alumnos, comparten lazos de amistad, militancia y formación.

La solidaridad en este contexto no es caridad o el humanitarismo de servicio social, sino que, en consecuencia, con la EP, puede entenderse como un conjunto de sentimientos y acciones compartidas que se organizan para crear esperanza y resistencia política, en el marco del compromiso por la justicia social. Una experiencia que da cuenta de ello es Lopes (2017), quien estudia la autoorganización de mujeres feministas de clases populares para la construcción de un preuniversitario popular alternativo, basado en la EP y una pedagogía feminista

É importante destacar que ela não é uma solidariedade fruto de caridade ou bondade, mas é marcadamente uma solidariedade política, por isso localiza-se no feminismo, e não apenas no gênero mulher. Este conceito e esta prática estão referenciados em uma consciência crítica sobre a origem e os princípios da misoginia, de articular e organizar coletivamente para romper com esta supremacia patriarcal desde a subjetividade até a cultura que, em níveis diferentes, ataca todas as mulheres. ( $p$. 70).

Como se evidencia, la solidaridad es una práctica de organización política de estos movimientos sociales juveniles y que rompe con la enseñanza bancaria. Aunque la solidaridad permite articular personas para trabajar juntas, en muchos casos bajo la amistad, ella no significa homogeneización. En todas las investigaciones consultadas confluyen distintos profesores y estudiantes que no comparten las mismas trayectorias académicas, pedagógicas y políticas, y por eso no es extraño que haya tensiones a lo largo de sus procesos.

Pero, ¿cómo la dialogicidad y solidaridad freiriana logran materializarse en los curriculum de estos proyectos educativos de movimientos sociales de jóvenes para una educación emancipatoria? una de las respuestas es que el

Campo da Educação Popular o currículo ultrapassa os limites disciplinares centrando-se em temas geradores, bem como maneja referencial teórico, conceitos, procedimentos e habilidades de diferentes áreas do conhecimento para solucionar as questões/problemas presentes no cotidiano dos educandos. (MORAIS, 2016, p. 48).

Morais (2016), quien a partir de un estudio de caso en Río de Janeiro estudia la construcción curricular de un PyP, explica que la inter/trans disciplinariedad son una de las apuestas más significativas. Vale la pena destacar que la inter/trans no es solamente un modo de construir conocimiento, es decir, un diálogo de las disciplinas, sino que también es una apuesta pedagógica-política por hacer un currículum distinto. en una racionalidad más 
relacional y compleja, con una serie de implicaciones políticas. Asimismo, la investigación de Santos, Gomes y Ferreira (2017), coincide en este punto, quienes investigan los PyP de la Universidade Federal de Uberlandia, y afirman que la interdisciplinariedad es el eje pedagógico más importante que orienta la construcción de materiales didácticos y los contenidos del curso.

Pero, ¿por qué puede ser política la inter/trans disciplinariedad? en primer lugar, se rompe con la supremacía de algunos campos de conocimiento sobre otros, por lo tanto, romper las divisiones y jerarquías crea un escenario distinto de diálogo, el mundo ya no es dual, no están las ciencias exactas y naturales por un lado y las sociales y humanas por otro, sino que ambas comparten problemas reconociendo sus especificidades; este rompimiento también es una ruptura en el lenguaje y las codificaciones de los saberes, y por ello un cambio en los valores, en cuanto que ya no hay una lógica binaria y secuencial, se pasa de la reproducción de conocimientos al debate y complementariedad.

En segundo lugar, se abre un nuevo campo: los saberes populares, ancestrales y culturales que traen consigo los estudiantes y sus contextos. En este sentido, las imágenes, las historias orales y la cosmogonía de los pueblos originarios de América Latina hacen parte de la enseñanza y el aprendizaje. Así pues, emergen saberes que anteriormente habían sido excluidos de las escuelas y las universidades en una nueva mirada más democrática y justa de los conocimientos y las culturas.

Al respecto, Martins (2018), quien investiga la formación en un PyP ubicado en Porto Alegre a través del análisis de las trayectorias escolares de sus profesores, afirma que

É através dessas iniciativas de ações e práticas democráticas que se constrói a mudança social. Os sujeitos envolvidos em espaços de Educação Popular têm o compromisso e o dever, no meu entendimento, de se posicionar a favor do protagonismo dos saberes leigos, tradicionais, urbanos, camponeses ou mesmo provindos da cultura não ocidental, como indígenas, africanos e orientais, segundo a ecologia de saberes de Santos. (p. 57).

Para la autora, los PP reflejan en diversos aspectos los legados del pensamiento freiriano, la pedagogía social y las pedagogías críticas de América Latina. En este contexto, la prueba de acceso no es la única finalidad de los PP, ya que en ellos se busca visibilizar más saberes que los académicos de las pruebas.

Para Martins, los PP son un compromiso de la responsabilidad social de la universidad. Por esto, muchos de los PP se articulan a proyectos de extensión universitarios y dejan de ser únicamente espacios de movimientos sociales a los bordes de las universidades. Es decir, si la docencia es la génesis de la 
universidad, la investigación su identidad, entonces la extensión es la que le da legitimidad, y una forma de legitimar es acercar a las clases populares a través de los PP, en la medida que la extensión es una herramienta de compromiso social. La autora explica que los PP nacieron al margen de la institucionalidad pero que a principios del siglo XXI muchos de ellos fueron institucionalizados.

Pero, ¿cuáles son las ventajas que un PP se institucionalice como un programa de la universidad?, ¿acaso esto no le restaría autonomía a un movimiento social y su propuesta de educación política? Para la autora, la universidad les ofrece no sólo recursos para asegurar su funcionamiento, sino plataformas institucionales para el diálogo y la formación de sus maestros, además de materiales tecnológicos. Las universidades apoyan procesos de selección de los profesores con el apoyo de practicantes y profesores asesores, y ofrece mejores condiciones físicas, y ocasionalmente algunas bolsas de apoyo económico. No obstante, se respeta la autonomía y libertad para la enseñanza y organización.

En este panorama, es importante insistir que cada PP tienen concepciones y prácticas diferentes de la EP, y por eso su institucionalización no ocurre igual. Mientras que en algunas universidades apenas se ofrece algunos apoyos pedagógicos y de recursos, en otras se vuelve hasta su principal agente dinamizador. No obstante, siempre los PP surgen a partir de las voluntades de estudiantes y profesores que si bien son agentes universitarios hacen parte de los movimientos estudiantiles y sindicatos profesorales

Por esto, no es de extrañar que muchos PP surjan a partir de las universidades, especialmente en el programa Conexiones de Saberes del MEC que surgió en el año 2006. Cabe señalar que la EP en Brasil puede ser pensada incluso como una referencia de políticas educativas, por ejemplo, con el Marco de Referência da Educação Popular para as Políticas Públicas del 2014 creado en el gobierno de Dilma.

Sin embargo, así como hay PP institucionalizados hay otros ligados exclusivamente a movimientos populares, como en el estudio de Lopes (2017), el cual muestra la relación de educación popular con el feminismo. Además, también aparecen recientemente dos estudios más que articulan las perspectivas decoloniales, especialmente con epistemologías de las poblaciones negras de Brasil con la educación popular para la preparación al examen de admisión a las universidades. Por un lado, Riberio (2018) en Río de Janeiro, y por otro Santos (2018) en Salvador de Bahia.

En ambas experiencias se crean PP para lograr el acceso de la población específicamente negra a la universidad como educación no formal y no institucional. De ambas disertaciones es interesante destacar el carácter colectivo y de lucha política que tienen sus miembros, en la medida que participan y actúan colectivamente. Además, suelen tener espacios de encuentro 
fuera de las salas de clase y crear proyectos comunitarios que envuelven a sus familias teniendo como centro de acción y reflexión la raza. Adicionalmente, en sus diseños curriculares suele estar presente la disciplina de ciudadanía y cultura, que como analiza Gomes (2015), como estudio de caso de un PP en Río de Janeiro, es una manera de mostrar la resistencia a las desigualdades sociales, puesto que en este espacio se discute sobre el capitalismo y la blanquitud en el contexto brasileño.

\section{Las universidades populares: teorías y prácticas}

La Educación popular, sus educadores, activistas e intelectuales han dejado como su único espacio de trabajo los centros de alfabetización o de educación de jóvenes y adultos para hacer parte de diversas agendas educativas, entre ellas, las universidades. Por esto, en las investigaciones es recurrente encontrar el concepto de universidad popular, describiendo algunas teorías y prácticas en torno a ella.

Pero, ¿qué es una universidad popular?, ¿cómo ocurren los procesos de selección y acceso a estas instituciones?, ¿de qué manera debe ser la docencia, investigación y extensión para que sea popular? y acaso ¿es posible que nuestras universidades sean populares? para pensar estas preguntas, todas las investigaciones sobre el tema coinciden en dos puntos: la primera es que las universidades están hechas por modelos europeos y norteamericanos que no responden a nuestras necesidades latinoamericanas, y el segundo es que la universidad como campo social está ligado indiscutiblemente a muchas de las lógicas y racionalidades del capitalismo y el neoliberalismo.

Ahora bien, si la universidad es una institución moderna, occidental y capitalista, y gran parte de lo que ocurre en ella responde a procesos basados en la meritocracia, la producción, la competencia, las exigencias de la economía, y a procesos de selección, clasificación y exclusión, que reducen los significados de lo público. En este panorama, Caride (2016) se pregunta cómo la educación popular y la pedagogía social pueden problematizar este panorama dentro de las mismas universidades en América Latina. Al respecto, uno de los conceptos freirianos más potentes que el autor resalta es el del diálogo

Las universidades construyen sus aprendizajes en el diálogo, al menos en una triple perspectiva: de un lado, el que establecen consigo mismas, en las comunidades docentes-discentes que articulan, institucionalmente, sus iniciativas científicas y académicas; de otro, el que tejen con la sociedad, haciendo uso de diferentes mecanismos de interacción y comunicación social, entre los que ocupan un lugar prioritario la extensión universitaria [...] finalmente, completando sus opciones, el que construyen los 
saberes disciplinares, abriéndose a los valores del pensamiento y del conocimiento fronterizo [...]. (CARIDE, 2016, s.p).

El diálogo como práctica social es uno de los desafíos de las universidades. Vale la pena aclarar que dialogar es una apuesta institucional para democratizar la universidad explorando en profundidad diversos sentidos de los diálogos cada vez más plurales, participativos y problematizadores. Sin embargo, las universidades no están hechas para el diálogo. Por un lado, por su estructura disciplinar, en donde los conocimientos no se relacionan y existen jerarquías entre ellos, excluyendo muchos de los saberes populares que son invisibilizados; por otro lado, las relaciones entre las disciplinas con el mercado exigen a la universidad ser evaluada en lógicas estrictamente económicas y de utilidades, por ende, se genera una estructura burocrática que no escucha y conversa, en la cual es más importante los formatos que los procesos; además, las pruebas de acceso y la producción y socialización del conocimiento suelen ocurrir entre pequeñas élites blancas urbanas (pequeña burguesía); adicionalmente, que la educación superior es un campo social donde es frecuente el fracaso y la expulsión sin escuchar a los que están a las márgenes de la institución. Finalmente, como diría Freire, una conversación no es una conversación si sólo transforma a una de las partes, y al parecer, la universidad se resiste a transformarse radicalmente.

En esta perspectiva, Paulo (2018), investiga cuáles son las concepciones sobre las universidades que los educadores populares e intelectuales cercanos a Freire tenían, o de las primeras generaciones de freirianos que aún están vivos. Entre sus conclusiones, Paulo muestra que la EP se ha ligado desde el comienzo de su historia a la lucha por la educación pública y los movimientos sociales, y que para los freirianos pensar en una universidad popular puede tener varios sentidos como: (a) políticas de acceso flexibles, amplias y democráticas, (b) conjunto de prácticas y políticas de permanencia que implican una reestructuración curricular, en términos democráticos en donde la educación es un derecho humano (c) prácticas pedagógicas y formas de investigación y extensión comprometidas con la clase trabajadora, legitimando los saberes hechos en Latinoamerica. Generalmente la EP dentro de las universidades hace mayor presencia a través de proyectos de extensión, y después aparece en docencia e investigación. Un ejemplo de esto está en Pereira y Gomes (2018), quienes explican cómo muchos PP surgen en programas de extensión universitario, y estos desde la extensión luchan por transformar desde adentro algunas estructuras universitarias para una construir una universidad popular.

En esta postura freiriana, la universidad popular no se limita al hecho de aceptar más clases populares y trabajadoras "pois uma coisa é abrir a universidade a pessoas do povo para inculcar também nelas saberes e valores hegemônicos; e outra coisa, bem diversa, é criar universidades envolvidas com contra-saberes e contravalores hegemônicos" (PAULO, 2010, p. 24). En vista de 
lo anterior, una universidad popular puede ser pensada como una contrauniversidad, en la medida que se resiste a través de múltiples tácticas a los modelos tradicionales que conocemos.

Ahora bien, esta contra-universidad no es tampoco una institución modelo o una forma administrativa concreta que se contenga en algún documento. No es posible encontrar registros que digan el paso a paso o los deberes de una universidad para que sea llamada "popular", y que contenga orientaciones para su fundación. Paulo muestra que no existe una matriz para medir exactamente qué tanto es una universidad popular o no. Lo popular no es tampoco una característica o dimensión de la universidad concreta, más bien, lo popular se refiere a un amplio conjunto de apuestas pedagógicas, políticas y éticas que se crean en las universidades para superar sus modelos contradictorios o insuficientes para la clase trabajadora. En definitiva, son proyectos en construcción que hacen de lo imposible lo inédito-viable en perspectiva freiriana, desde la docencia, investigación y extensión.

Todo ello significa que hay universidades que si bien son fundadas en modelos tradicionales ellas apuestan por ser populares, u otras que surgen con la perspectiva popular. Hay múltiples experiencias al respecto, por ejemplo, Rebua (2015) analiza dos casos, uno en Argentina y otro en Brasil, específicamente la Escola Nacional Florestan Fernandes UNFF (SP) y la Universidad Popular de Madres de Plaza de Mayo (UPMPM). Ambas surgen por movimientos sociales y como alternativas a las crisis del neoliberalismo, funcionan como plataformas de formación política y empoderamiento de campesinos y mujeres, en asocio con universidades tradicionales, pero que se diferencian en diversos aspectos unas de otras. Por ejemplo, Morris (2017), analiza las propuestas de la Universidad Popular de los Movimientos Sociales y señala que uno de los puntos principales de la propuesta era superar el colonialismo académico o "prática extrativista do conhecimento" (p.23), ya que una universidad popular trabaja con los saberes ancestrales y marginados de las universidades a través de la interculturalidad.

Otros casos son los de las universidades federales multicampi en Fronteira Sul (UFFS), con sede en Chapecó, Santa Catarina, y la Universidad del Sul de Bahía (UFSB), con sede en Itabuna, Bahía, que como afirman Santos y Tavares (2016), son universidades populares en cuanto a se comprometen institucionalmente con la inclusión de epistemologías, territorios y sujetos históricamente excluidos. Por ejemplo, para el caso de la UFSB, Friggeri y Guanaes (2017), muestran que lo popular se extiende a comprensiones más políticas con las transformaciones sociales en el caso de proyectos de extensión y investigación. Una universidad que se destaca en esta misma lógica es la de Universidade da Integração Internacional da Lusofonia Luso Afro brasileira (UNILAB), como lo afirman (GOMES, VIEIRA, 2013). 
También aparecen experiencias de universidades convencionales pero que toman elementos de la EP como principios y horizontes institucionales, es el caso de la Unisinos, que como expresa Streck y Rosa (2015), busca comprometerse con las clases populares reconectando sus necesidades con la universidad. De esta manera, los autores retoman las ideas de universidad popular de José Carlos Mariátegui y José Martí, ellos explican que dos elementos importantes de una universidad para que sea realmente popular es que se problematice e investigue la historia de los pueblos americanos, se valorice las culturas populares de nuestra región, se superen las dicotomías entre saberes legítimos y otros invisibilizados, y se formen líderes latinoamericanos para gobernar a nuestros pueblos sin una mentalidad europea.

Al respecto, se destaca dentro de los antecedentes los aportes de la Revista Lusófona de Educação, n. 24, en su Dossier Paulo Freire e a Educação Superior. En el dossier, en el artículo de Tavares (2013), se dice que una universidad popular es aquella que problematiza y profundiza sobre el significado de la democracia dentro de sí misma. Tavares toma los aportes de Boaventura de Sousa Santos para afirmar que una universidad popular es

Intertemática, ou seja, o seu caráter intertemático reside na
promoção de reflexões e articulações entre a diversidade de
movimentos (operários, feministas, indígenas,
afrodescendentes, ecológicos) de modo a criar no mundo um
movimento e ação progressistas e uma nova consciência
internacionalista que seja intercultural e radicalmente
democrática. (p. 157).

En este sentido, la universidad popular trabaja con la sociología de las ausencias, como lo estudia Boaventura de Santos, y alcanza una ecología de saberes. Para que esto sea posible, Romão (2013) aborda el concepto de concientización de Freire, discutiendo cuáles son las responsabilidades de los intelectuales contemporáneos. Para este investigador, las universidades populares tienen matrices institucionales en búsqueda de la superación de la lógica de mercado y en contraposición a la matriz neoliberal, él entiende las universidades en dos sentidos: un compromiso con la igualdad (acceso) pero también con su transformación institucional para superar su lógica empresarial.

Para terminar, ¿cuáles son las experiencias de los PP en Colombia y Medellín? y ¿cómo estos resultados conversan con los de Brasil?

\section{¡palabras finales...voces de esperanza!}

Diversos preuniversitarios en Colomboa y la ciudad de Medellín aparecen en las redes sociales y académicas mostrando su trabajo. Algunos de estos 
espacios alternativos de educación pertenecen a organizaciones sociales y políticas, y otros funcionan de modo independiente, lo que da cuenta del pluralismo que hay entre ellos. Se resaltan en la búsqueda realizada en Medellín los PP Papalotl, Barriada Popular, Colectivo de Educación Popular Paulo Freire, Barricadas de Papel, Abya Yala, Grupo AEQUUS, Benkos Biohó, entre otros. En esta búsqueda gran parte de la información no está en fuentes de información institucionales, sino en de lo que comúnmente se conoce como deep web, o en sitios virtuales alternos como blogs, asociaciones de estudiantes y profesores y presentaciones elaboradas para eventos con acceso abierto.

Cabe resaltar que, a diferencia de Brasil, en donde los PP constituyen un movimiento institucionalizado de muchas universidades y con una trayectoria amplia de trabajo que repercute en políticas afirmativas, además, con un número considerable de investigaciones de maestría y doctorado, en Colombia el panorama es bastante diferente. En primer lugar, los PyP no son programas universitarios, al igual que en Brasil en sus comienzos, son propuestas ques se gestan y desarrolllan a los bordes de las universidades, y que operan de manera autónoma como educación no formal. Incluso la mayoría de estos grupos no trabajan con la universidad, en la medida que las administraciones universitarias son vistas como reproductoras de las desigualdades sociales a las que combaten, contrario a los principios de la EP.

En segundo lugar, los PP en Colombia han sido parte y dinamizadores de diversas manifestaciones y paros estudiantiles dentro de los movimientos y asambleas estudiantiles, ellos han discutido en varios escenarios con los decisores de políticas educativas el acceso a la universidad, pero no han constituido, como en Brasil, bases para políticas afirmativas. La mayoría de material que hay sobre ellos no son objetos de conocimiento de disertaciones y tesis de maestría y doctorado, ya que casi todo lo que hay escrito no está en medios académicos oficiales, sino que está en encuentros de educación popular, blogs y unos cuantos trabajos publicados.

Esta diferencia entre ambos países puede explicarse de diversos modos, en la medida que la historia de la universidad, los gobiernos y las políticas educativas de Brasil y Colombia son muy diferentes, al igual que los movimientos estudiantiles. Las maneras de hacerse efectivo el neoliberalismo y las luchas contra él si bien coinciden y dialogan en algunos puntos, ellas no son iguales. Si bien en Colombia los PP no son institucionalizados y son raras las investigaciones al respecto, ellos no son grupos menores, ya que el número de estudiantes que participan de los PP en el país supera los 10.000, con más de 30 experiencias en el territorio nacional, como se menciona en el VI Encuentro de Educación Popular realizado en la Universidad Pedagógica Nacional durante el mes de septiembre de 2019.

El más antiguo de todos estos preuniversitarios en la ciudad de Medellín es Papalote (PAPALOTE EQUIPO, 2011, 2013), (MONTOYA, 2018), el cual 
inicia en la década de los 90 en la Universidad Nacional de Colombia sede Medellín. En las historias y recuentos de experiencia se muestra que sus trabajos si bien comenzaron por una coyuntura política por el cambio de admisión en las universidades colombianas en 1993, sus luchas son estructurales. Es decir, ellos se plantean la relación universidad, ciudad, Estado y democracia como elementos claves para la transformación social.

Las lógicas neoliberales que privatizaron y siguen privatizando las universidades son amenazas constantes que se han mantenido a lo largo del tiempo y por eso el preuniversitario continua. Los estudiantes se preparan para acceder a la Universidad Nacional (sede Medellín) y la Universidad de Antioquia. Ellos enseñan clases de lengua castellana, comprensión lectora, matemáticas y razonamiento lógico, además de un tercer espacio formativo llamado contexto, en el cual analizan temas como la memoria y la identidad de los barrios populares y los lugares de los que vienen sus estudiantes. A lo largo de sus cerca de 20 años de existencia han vivido diferentes transformaciones, en las cuales ha sido fundamental para mantener los procesos la concepción de la unión y el colectivo para potenciar sus prácticas de resistencia.

Por otro lado, el Preuniversitario Popular Barriada surge "por la elitización de las universidades en colombia y la dificultad de los jóvenes de colegio públicos para estudiar em la universidad. Es una resistencia a eso" (Preuniversitarios populares, 2014). En este espacio se trabaja con talleres temáticos de lectura, escritura y matemáticas, además de otros alternos sobre el territorio, el derecho a la ciudad y el género desde una perspectiva social y crítica. Al igual que en Papalot para los educadores populares de este proyecto es importante superar el egoísmo que caracteriza las relaciones humanas actuales y educativas, por otra solidaria y reflexiva.

En este mismo sentido, el Colectivo de Educación Popular Paulo Freire "surge como lo bueno, a partir de la amistad. Nace por estudiantes de la Universidad de Antioquia que sentían la necesidad y responsabilidad como jóvenes con oportunidades para dar oportunidades a otros jóvenes" (Preuniversitarios populares, 2014). En este preuniversitario se preguntan y problematizan por el número y los perfiles de poblaciones que ingresan a la universidad, apostando para que los campesinos e hijos de obreros accedan a la educación superior. Ellos se han asociado con distintas escuelas, corporaciones y casas culturales y han expandido su trabajo a talleres formativos comunitarios en barrios populares de la ciudad de Medellín.

Otro escenario alternativo de educación es el que se desarrolla en Itagui, área cercana de Medellín, por el grupo AEQUUS (OSPINA, AMP; ZULUAGA, 2015). Inicialmente este grupo funcionaba únicamente como un preuniversitario, en un contexto difícil por estar insertos en un barrio violento donde circulaban distintas redes de tráfico y delitos que ponían a su juventud al servicio del crimen. No obstante, el preuniversitario logró extenderse a otras prácticas educativas 
para la comunidad como son las clases de literatura y baile, de hecho, es interesante en este proceso ver cómo la comunidad se empodera de las acciones del grupo y una práctica genera otras prácticas, y una persona dinamiza y transforma a muchas.

De estas experiencias queremos resaltar varios aspectos. Uno de ellos es que se supera el trabajo exclusivamente académico de los preuniversitarios tradicionales. Esta razgo trae consigo una nueva visión: para ser universitario y hacer uso del derecho a la educación superior gratuita y de calidad no se necesita únicamente habilidades cognitivas y de aprendizaje relacionadas a la calidad educativa, sino también una visión crítica de lo que implica estudiar en universidad pública, en un país con amplias desigualdades sociales.

Este cambio de lo exclusivamente académico a lo político y ético promueve a concebir de una manera distinta al estudiante y la universidad, ya no en términos de contenidos, sino de actitudes y pensamientos enmarcados en contextos sociales y culturales determinados que necesitan ser transformados. Esta mirada problematizadora, transformadora y crítica que los PP apropian de Paulo Freire y la educación popular pone de relieve las luchas históricas de las universidades y sus movimientos por resistir a su desaparición. Asimismo, se procura una comprensión mayor de las desigualdades sociales. En consecuencia, la EP otorga de sentido crítico y social los espacios universitarios, inspirados en el legado de Paulo Freire.

Otro aspecto es el concepto de resistencia que es bastante frecuente en el caso colombiano. Ejemplos de ello son el trabajo de Mora y Ortiz (2016) y Serrano (2016), quienes sistematizan las experiencias de dos PP en Bogotá. Para estos investigadores, los PP son organizaciones juveniles, que dan cuenta de la participación política y democrática de las nuevas generaciones donde ocurren procedimientos de socialización que forman las identidades de los grupos y sujetos participantes.

De todas las experiencias resaltadas tanto en Medellín (Colombia) como en Brasil hay elementos comunes que si bien difieren en posiciones y posturas caracterizan a estos espacios de formación. A continuación, se presentan esos elementos como una conclusión y que permiten a partir de toda la lectura documental caracterizar a los PP

- Las prácticas de los PyP surgen y se desarrollan en el marco de posturas políticas explícitas, esto significa que reconocen la politicidad de la educación. Ahora bien, este horizonte de los PP hace parte en una serie heterogénea de movimientos sociales, sindicatos y colectivos de estudiantes y profesores que abarcan distintas y diferentes pensamientos políticos. En este sentido, constituyen una amalgama de multiples pensamientos que, en general, se mueven en el amplio espectro de la izquierda latinoamericana. 
- Los PP son espacios de formación de jóvenes y para jóvenes. En la historia de sus colectivos un rasgo característico es la participación activa de los jóvenes, ya sea como profesores o estudiantes. En todas las experiencias el movimiento estudiantil tanto de colegios como de las universidades públicas y privadas es un actor fundamental para fundar e impulsar los PP.

- En los PP, por lo popular se hace un recorte principalmente de clase, corresponde aquellos segmentos de la población que no pertenecen a las clases sociales altas, las diferentes burocracias de las clases medias o conjuntos de la población que tradicionalmente tienen el poder político y económico, y por eso mismo privilegios culturales y sociales. De este modo, lo popular son las personas y comunidades que han sido histórica y culturalmente excluidas, especialmente por las desigualdades sociales del país. Para los antecedentes y problematización de esta investigación en los PP que se indican se reconoce como popular a los que viven en las periferias de las ciudades, es decir, en las márgenes de los lugares donde están las clases sociales altas o medias.

- En el interior de sus grupos, entre profesores y estudiantes se desarrollan relaciones humanas más cercanas que las laborales o académicas tradicionales de cualquier institución educativa.

- La lucha contra el neoliberalismo basados en la esperanza, el diálogo de saberes y la democracia.

Estos elementos de diferencia y semejanza entre los PP de Brasil y Colombia dan cuenta de caminos posibles para futuras investigaciones comparadas que busquen comprender cómo la EP es apropiada y puesta en marcha en diferentes contextos de América Latina. Por último, es una muestra de que el pensamiento de Paulo Freire no es estatico, ya que adquiere en diversas geografías de nuestra región matices diferentes, pero que en todo caso sigue animando investigaciones y practicas educativas de jóvenes, que basados en la esperanza, problematización y solidaridad Freiriana genera una diversidad amplia y compleja de proyectos e iniciativas que necesitan articularse cada vez más entre ellas y las universidades.

\section{REFERENCIAS}

CARIDE, José A. La pedagogía social en el diálogo de las universidades con la educación popular y la educación social. Revista Interamericana de Educación de Adultos, México DF, vol. 38, núm. 1: enero-junio, 2016. Disponible en: https://www.redalyc.org/jatsRepo/4575/457545337006/html/index.html.

SOUSA, Israel de. Educação popular e ensino de história local: cruzando conceitos e práticas. João Pessoa: UPFB, 2015. Disponible em:

https://sucupira.capes.gov.br/sucupira/public/consultas/coleta/trabalhoConclusao/view TrabalhoConclusao.jsf?popup=true\&id trabalho=3446938. 
SANTOS, A. Gomes, G. Ferreira, S. Integrated Formative Actions (AFIN): results and challenges of preparatory course for admission to higher education at the Federal University of Uberlândia. Revista de Educação Popular. Uberlandia, Vol 16, Iss 3: 2017. Disponible en:

https://www.ingentaconnect.com/content/doaj/16785622/2017/00000016/00000003/art 00008;jsessionid=7j7yxqdhjabq. $x$-ic-live-02.

SANTOS, Barbara C. dos O Ensino de Química em um Curso de Educação Popular preparatório para o ENEM. Pelotas: UFPEL, 2017. Disponible en: https://sucupira.capes.gov.br/sucupira/public/consultas/coleta/trabalhoConclusao/view TrabalhoConclusao.jsf?popup=true\&id trabalho=5195817.

IV Encuentro de la Educación Popular - Día 2. Producción de la Universidad Pedagógica Nacional. Bogotá: Auditorio Simón Rodríguez. 2019. Videograbación (3:27:20). Disponible en: https://www.youtube.com/watch?v=Tvbl23kQ02.

FALEIRO, F. Farias, M. Inclusion of peasant women in university: among dreams, challenges and struggles. Revista Educação e Pesquisa. São Paulo, Vol, 43, núm 3: 2017. Disponible: http://www.scielo.br/scielo.php?pid=S1517$\underline{97022017000300833 \& \text { script }=\text { sci arttext\&tlng=en. }}$

FRIGERI, P. GUANAES, A. Universidade popular e democratização de saberes: o caso da universidade federal do Sul de Bahia. Revista Eccos - Revista Científica. São Paulo, jan-abr 2017, Issue 42: 2017. Disponible en: https://www.redalyc.org/articulo.oa?id=71550676004.

GOMES, N. VIEIRA, S. Construindo uma ponte Brasil-África: a Universidade da Integração Internacional da Lusofonia Luso- Afro-Brasileira (UNILAB). Revista Lusófona de Educação. Lisboa, N. 24, 2013. Disponible en: https://revistas.ulusofona.pt/index.php/rleducacao/issue/view/632.

GOMES, Renato. A formação do PVNC - Núcleo Vila Operária: uma experiência de inclusão educacional. Rio de Janeiro: PUC, 2015. Disponible en: https://sucupira.capes.gov.br/sucupira/public/consultas/coleta/trabalhoConclusao/view TrabalhoConclusao.jsf?popup=true\&id trabalho=3491083.

KESLEY RAMOS, Kesley. Desafios políticos ao currículo de inglês em uma perspectiva decolonial: um estudo no Pré-Vestibular Comunitário Saber para Mudar em 2014 e 2015. Rio de Janeiro: UERJ, 2018. Disponible en:

https://sucupira.capes.gov.br/sucupira/public/consultas/coleta/trabalhoConclusao/view TrabalhoConclusao.jsf?popup=true\&id trabalho=6497955.

LOPES, Daniele. Quem não pode com a formiga não atiça o formigueiro: a autoorganização das mulheres e a educação popular na construção da pedagogia feminista no curso Desario Pré-vestibulares. Pelotas: UFPEL, 2017. Disponible en: https://sucupira.capes.gov.br/sucupira/public/consultas/coleta/trabalhoConclusao/view TrabalhoConclusao.jsf?popup=true\&id trabalho $=5584378$.

MARTINS, Maria E. Universidade e Comunidade: A experiência do pré-vestibular esperança popular da restinga e a formação humana. Porto Alegre: UFRGS, 2018. Disponible en:

https://sucupira.capes.gov.br/sucupira/public/consultas/coleta/trabalhoConclusao/view TrabalhoConclusao.jsf?popup=true\&id trabalho=629880. 
MONJANE, B. Lições da Universidade Popular dos Movimentos Sociais na África Austral: terra, luta e emancipação. Revista da sociedade de pesquisa qualitativa em motricidade humana. São Paulo, Vol 2, núm 2: 2018. Disponible en: http://www.motricidades.org/journal/index.php/journal/article/view/25946463.2018.v2.n2.p163-174.

MONTOYA, R. Análisis de las prácticas del Proyecto Papalotl a partir de la experiencia "Diarios de Contexto" en el marco de los postulados Educación Popular Proyecto Aplicado. Medellín: Universidad Nacional Abierta y a Distancia. 2018. Disponible en:

https://stadium.unad.edu.co/preview/UNAD.php?url=/bitstream/10596/21195/1/717799 13.pdf.

MORA, Y. ORTIZ, A. Sistematización de la experiencia educativa y popular del colectivo Libremente. La configuración de una prácticas pedagógica, política y organizativa. Bogotá: Universidad Pedagógica Nacional, 2016. Disponible en: http://repositorio.pedagogica.edu.co/bitstream/handle/20.500.12209/689/TO19938.pdf?sequence $=1$ \&isAllowed $=\mathrm{y}$.

MORAIS, Adrielly. Curso pré-vestibular popular do Centro de Estudos e Ações Solidárias da Maré CEASM: desafios múltiplos na confluência de práticas curriculares. Rio de Janeiro: UFF, 2016. Disponible en:

https://sucupira.capes.gov.br/sucupira/public/consultas/coleta/trabalhoConclusao/view TrabalhoConclusao.jsf?popup=true\&id trabalho=3621710.

MORRIS, E. Una mirada a la educación popular y las epistemologías del Sur: la Universidad Popular de los Movimientos Sociales. Revista Estudios de filosofía práctica e historia de las ideas. Mendoza, Vol 19, núm 1: 2017. Disponible en: https://dialnet.unirioja.es/servlet/articulo?codigo=6697214.

OSPINA, C. \& ZULUAGA, D. Sistematización de las experiencias de los jóvenes del grupo AEQUUS que han posibilitado en su práctica socioeducativa el compromiso social frente a la formación académica de otros jóvenes, entre los años 2010. 2013. Medellín: Universidad de Antioquia, 2015. Disponible en: http://200.24.17.74:8080/jspui/bitstream/fcsh/279/1/OspinaCatalina sistematizacionexp erienciasjovenesgrupoaequusposibilitadopracticasocieducativacompromisosocialforma cionacademica.pdf.

PAULO, F. Pioneiros e pioneiras da Educação Popular freireana e a universidade. São Leopoldo: UNISINOS, 2018. Disponible en:

https://sucupira.capes.gov.br/sucupira/public/consultas/coleta/trabalhoConclusao/view TrabalhoConclusao.jsf?popup=true\&id trabalho=6242008.

PAPALOTE EQUIPO. ¿Qué es eso de Papalotl? Revista Kavilando, N. 2 VOL 2, 2011. Disponible en: https://nbn-resolving.org/urn:nbn:de:0168-ssoar-429532.

PEREIRA, V. Gonçalves, L (orgs). Educação popular no contexto do PAIETS - FURG: os saberes da pesquisa em extensão universitária. Rio Grande: FURG, 2012.

PEREIRA, V. Gonçalves, L (orgs). Ventos que sopram no Sul: vivências de educação popular e de transformação social no PAIETS. Rio Grande: FURG, 2012.

PEREIRA, T, GOMES, T, F. A extensão universitária em debate: o curso préuniversitário como espaço de educação popular. Revista Espaço Pedagógico, Passo 
Fundo, Vol 25, Iss 3: 2018. Disponible en:

http://seer.upf.br/index.php/rep/article/view/8570.

Preuniversitarios Populares de Medellín y áreas cercanas. Producción de

Preuniversitarios Populares. Medellín: $1^{\circ}$ Encuentro de Preuniversitarios Populares de Medellín y áreas cercanas realizado los días 8 y 9 de Noviembre del año 2014. 2014. Videograbación Disponible en: https://www.youtube.com/watch?v=ueWRhzdomww.

Preuniversitarios Populares de Medellín y áreas cercanas. Producción de Esteban Marín. Medellín: $1^{\circ}$ Encuentro de Preuniversitarios Populares de Medellín y áreas cercanas realizado los días 8 y 9 de Noviembre del año 2014. 2014. Videograbación Disponible en: https://www.youtube.com/watch?v=g9k84vomshY.

PROYECTO PAPALOT. Proyecto Papalotl. en IV ENCUENTRO NACIONAL E INTERNACIONAL Y VIII REGIONAL DE EXPERIENCIAS EN EDUCACIÓN POPULAR Y COMUNITARIA, octubre, 2013.

REBUÁ, CARLOS, E. Da praça ao solo: um novo chão para a universidades: As experiências das universidades populares de Madres de Plaza de Mayo [UPMPM] e Movimento dos Trabalhadores Rurais Sem Terra [ENFF] em tempos de crise neoliberal na América Latina, Rio de Janeiro: UFF, 2015. Disponible en: https://sucupira.capes.gov.br/sucupira/public/consultas/coleta/trabalhoConclusao/view TrabalhoConclusao.jsf?popup=true\&id trabalho=2988044.

RIBEIRO, Jorge A. Cursos Pré-vestibulares comunitários e jovens da zona oeste: 0 caso do IFHEP e sua contribuição para o combate do racismo. Rio de Janeiro: Centro Federal de Educação Tecn, 2018. Disponible en:

https://sucupira.capes.gov.br/sucupira/public/consultas/coleta/trabalhoConclusao/view TrabalhoConclusao.jsf?popup=true\&id trabalho=7060634.

ROMÃO, J. Paulo Freire e a Universidade. Revista Lusófona de Educação. Lisboa, N. 24, 2013. Disponible en:

https://revistas.ulusofona.pt/index.php/rleducacao/issue/view/632.

SANTOS, E. Tavares, M. Desafios Históricos da Inclusão: Características Institucionais de Duas Novas Universidades Federais Brasileiras. Revista Education Policy Analysis Archives. Arizona, Vol. 24 Issue 62/63: 2016. Disponible en: https://dialnet.unirioja.es/servlet/articulo?codigo $=5623067$.

SANTOS, Paulo R. História e ousadia. Resistência na periferia: o caso dos quilombos educacionais do Orobu. Salvador de Bahía: UNEB, 2018. Disponible en:

https://sucupira.capes.gov.br/sucupira/public/consultas/coleta/trabalhoConclusao/view TrabalhoConclusao.jsf?popup=true\&id trabalho=717114.

SILVA, Renán. Dos compromissos da geografia no pré-vestibular popular. Porto Alegre: UFRGS, 2015. Disponible en:

https://sucupira.capes.gov.br/sucupira/public/consultas/coleta/trabalhoConclusao/view TrabalhoConclusao.jsf?popup=true\&id trabalho=2623954.

SERRANO, K. Transformando el lugar de la educación. Organizaciones juveniles en Tunjuelito: cuatro escenarios de socialización y aprendizajes, Bogotá: Colegio Mayor de Nuestra Señora del Rosario, 2016. Disponible en:

https://core.ac.uk/download/pdf/86438528.pdf. 
STRECK, D. ROSA, C. Conexiones necesarias: la educación popular en la universidad. Revista La Piragua, Lima, N. 41, noviembre 2015. Disponible en: http://www.fahce.unlp.edu.ar/extension/Documentos\%20y\%20Ponencias/lainvestigacion-accion-participativa-entre-cs-soc-y-educ-popular.

TANGERINO, Stella. Experiências ao rés do chão - uma visão para a educação popular: estudo de caso sobre o cursinho popular Florestan Fernandes. São Paulo: USP, 2018. Disponible en:

https://sucupira.capes.gov.br/sucupira/public/consultas/coleta/trabalhoConclusao/view TrabalhoConclusao.jsf?popup=true\&id trabalho $=7037419$.

TAVARES, M. A Universidade e a pluridiversidade epistemológica: a construção do conhecimento em função de outros paradigmas epistemológicos não ocidental cêntricos. Revista Lusófona de Educação. Lisboa, N. 24, 2013. Disponible en: https://revistas.ulusofona.pt/index.php/rleducacao/issue/view/632.

VASCONCELOS, André. Pré-vestibulares populares: desafios políticos ao currículo e ensino de Geografia. Rio de Janeiro: UERJ, 2015. Disponible en:

https://sucupira.capes.gov.br/sucupira/public/consultas/coleta/trabalhoConclusao/view TrabalhoConclusao.jsf?popup=true\&id trabalho=3062103. 